Research Paper

\title{
Demethylzeylasteral inhibits cell proliferation and enhances cell chemosensitivity to 5 -fluorouracil in Colorectal Cancer cells
}

\author{
Yang Yang ${ }^{1^{*}}$, Jing $\mathrm{Han}^{2 *}$, Yanlin $\mathrm{Ma}^{3}$, Jianfeng Zhang ${ }^{1}$, Zhenya Zhang ${ }^{1 凶}$, Guiying Wang ${ }^{4,3 凶}$ \\ 1. Department of General Surgery, The Fourth Hospital of Hebei Medical University, Shijiazhuang, 050011, China. \\ 2. Department of Medical Oncology, The Fourth Hospital of Hebei Medical University, Shijiazhuang, 050011, China. \\ 3. The Fourth Hospital of Hebei Medical University, Shijiazhuang, 050011, China. \\ 4. The Third Hospital of Hebei Medical University, Shijiazhuang, 050011, China. \\ *These authors contribute equally in this work. \\ $\square$ Corresponding authors: Guiying Wang, The Third Hospital of Hebei Medical University/The Fourth Hospital of Hebei Medical University, Shijiazhuang, \\ 050011, China, E-mail: wangguiyingtgzy@163.com and, Zhenya Zhang.
}

(0) The author(s). This is an open access article distributed under the terms of the Creative Commons Attribution License (https://creativecommons.org/licenses/by/4.0/). See http://ivyspring.com/terms for full terms and conditions.

Received: 2020.01.30; Accepted: 2020.07.16; Published: 2020.08.19

\begin{abstract}
Malignant growth and chemotherapy resistance to 5-fluorouracil (5-FU) are the obstacles to the treatment of Colorectal cancer (CRC). There is need to develop effective therapeutic option. Demethylzeylasteral benefits to immune and anti-tumor function. However, the role demethylzeylasteral played in colorectal cancer remains unclear. Here, our study confirmed that demethylzeylasteral could inhibit the cell malignant capacity, such as proliferation, migration and invasion. And we also found demethylzeylasteral could cause cell cycle arrest and apoptosis. Followed we verified that combination demethylzeylasteral with 5-FU has a better curative effect in vitro. The two drugs function synergistically in SW480 and additionally in RKO. IC50 values of 5-FU decreased when combined with demethylzeylasteral. Next, we used the network pharmacology approach to explore the the potential molecular mechanism of demethylzeylasteral. We constructed the "Colorectal - targets Demethylzeylasteral" and protein-protein interactions (PPI) networks. And 15 hub genes were found in PPI network. Then Gene Ontology (GO) enrichment analysis showed that demethylzeylasteral may affect cell cycle, apoptosis, invasion and response to chemotherapy drugs. Kyoto Encyclopedia of Genes and Genomes (KEGG) pathway analysis indicated demethylzeylasteral may be involved in many cancerrelated pathways. Taken together, the network pharmacology approach provided a potential mechanism of demethylzeylasteral in colorectal cells. Our study indicated that demethylzeylasteral could exert antitumor effects and enhance the sensitivity of the Colorectal cells to 5-FU, suggesting a promising ability to serve as an anti-cancer agent in Colorectal cancer.
\end{abstract}

Key words: Colorectal, Demethylzeylasteral, 5-Fluorouracil, Chemosensitivity

\section{Introduction}

Colorectal cancer (CRC), one of the most common cancers worldwide, had caused a higher and higher morbidity and mortality each year [1-3]. It had been reported that about 900000 people died every year [4]. In China, 20.7 per 100,000 were projected to be diagnosed with colorectal in 2020 and the casefatality ratio in China was $14.0 \%$ [5]. The treatment for colorectal was an enormous challenge.

Unfortunately, those about $20 \%$ patients were in the middle and late period when see a doctor complicated the treatment [6]. Chemotherapy represented an essential treatment for patients who were in advanced stage [7]. 5-Fluorouracil (5-FU), playing a critical role in regulating the cell cycle and apoptosis [8], had emerged as the front-line chemotherapeutic drug for colorectal cancer [9]. However, many patients had no response to 5-FU, leading to recurrence or even worsened condition 
[10]. Chemotherapy resistance to 5-FU became an issue urgently needed to be solved.

Demethylzeylasteral originally was thought to have immunosuppressive and anti-inflammatory effects [11]. It was recently confirmed that demethylzeylasteral could inhibit angiogenesis as well as cell activity in many cancers, such as breast cancer, glioma, melanoma and so on [12-14]. Furthermore, recent study evidence showed that demethylzeylasteral could enhance chemosensitivity to gemcitabine in human pancreatic cancer cells [15].

However, the influence of demethylzeylasteral on colorectal cell has remained unclear. In our study, we indicated that demethylzeylasteral could suppress the cell proliferation, migration and invasion in a dose dependent manner. The study on enhancing chemosensitivity to 5-FU was also at the heart. Then, the binding targets of demethylzeylasteral were predicted to explore the mechanism. We got the intersection genes of binding targets and colorectal cancer-related genes. These genes were used to construct the "Colorectal - targets - Demethyl zeylasteral" and PPI networks. In addition, 15 hub genes in the PPI network had been found. Followed GO and KEGG pathway analyses revealed us the potential mechanism of Demethylzeylasteral. Our study showed that the combination demethylzeylasteral with 5-FU could achieve a better therapeutic effect.

\section{Materials and Methods}

\section{Cell Culture}

The RKO was maintained in RPMI-1640 complemented with $10 \%$ fetal bovine serum. The SW480 was complemented in DMEM (Dulbecco's Modified Eagle Medium) containing 10\% fetal bovine serum. All cell lines were cultured at $37^{\circ} \mathrm{C}$ in $5 \% \mathrm{CO} 2$. When the cells proliferated to $80 \%$ confluence, the cells were digested with trypsin, centrifuged, washed with aseptic PBS twice and plated on the cell petri dish. The cells in the logarithmic proliferative phase were taken for the experiment.

\section{Western Blotting analysis of protein}

Western Blotting assays were performed as previously described [16].

\section{Proliferation assay}

The cells were plated on 96 wells (5000 cells per well) when the cells were logarithmic phase. The absorbance was examined $0,24,48,72$ and $96 \mathrm{~h}$ after inoculation by MTS assay.

\section{Colony formation assay}

The cells were plated on 24 wells (20000 cells per well) when the cells were logarithmic phase. After 2 days, the cells were fixed with methanol for 5 minutes, stained with crystal violet for 5 minutes, and the number of cells was observed.

\section{Transwell analysis}

Cells were plated on upper chamber of transwell (300000 cells per well) when the cells were logarithmic phase. After 36 hours, the chamber was fixed in methanol for 5 minutes and stained with crystal violet for 5 minutes. The number of cells passing through Transwell chambers was observed under microscope.

\section{Cell cycle analysis}

After the cells were treated for the day ahead (centrifuged, washed, re- centrifuged, and fixed with $70 \%$ precooled ethanol), the cells were placed in the-20 refrigerator overnight. The next day, the cells were centrifuged, washed, centrifuged again, incubated in dark for 15 minutes with PI (BD Biosciences, San Jose, CA, USA) reagent and were detected by upflow cytometry.

\section{Apoptosis detection}

Cells were digested by trypsin, centrifuged, washed, centrifuged again and a series of operations are carried out according to the instructions of apoptosis kit (Neo Bioscience, Beijing, China). The cells were detected by upflow cytometry last.

\section{Combined effect analysis}

The drug-drug interaction coefficient (coefficient of drug interaction, CDI) was used to evaluate the drug-drug interaction [17]. If CDI $<1$, it was proved that the action of the two drugs was synergistic, and when CDI < 0.7, the synergistic effect of the two drugs was very significant; if $\mathrm{CDI}=1$, the action of the two drugs was additive; if CDI $>1$, the action of the two drugs was antagonistic.

\section{Data collection and processing}

The 3D molecular structure of demethylzeylasteral was got from PubChem database (https://pubchem.ncbi.nlm.nih.gov). The binding sites of demethylzeylasteral were predicted by PharmMapper database (http://www.lilab-ecust.cn/ pharmmapper/). The sequencing data of Colorectal normal tissues and tumor tissues were downloaded from The Cancer Genome Atlas database (TCGA, https://portal.gdc.cancer.gov/). The colorectal related genes and predicted binding genes of demethylzeylasteral were inputed into jvenn (http:/ / jvenn.toulouse.inra.fr/app/example.html) to achieve the intersection genes. The "Colorectal - targets Demethylzeylasteral" network was constructed by using Cytoscape software and PPI network was 
constructed by using STRING database (https:// string-db.org). The hub genes of PPI network were found by using Cytoscape software (according to the degree algorithm). The GO and KEGG pathway analyses were carried out by using DAVID 6.8 database (https://david.ncifcrf.gov). The Bubble charts were made by Rstudio 3.6.2 software. The cox regression analysis of the survival rate of these hub genes in CRC through Oncolnc database (http:// www.oncolnc.org/).

\section{Statistical analysis}

The experimental results were analyzed by Student's t-test (unpaired, two tailed). $P<0.05$ were considered to be significant. All statistical analyses were performed using Prism5 (GraphPad Software Inc., La Jolla, CA).

\section{Results}

\section{Demethylzeylasteral inhibited the colorectal cell malignancy}

To investigate whether Demethylzeylasteral affects the cell viability, we treated colorectal cells by demethylzeylasteral of gradient concentrations. Its molecular structure was shown in Figure 1A. We found that demethylzeylasteral inhibited the proliferation of colorectal cells, showing a dosedependent manner (Figure 1B). Then the MTS assays were used to verify this result. With the dosage increasing, the proliferation and growth of cells were inhibited (Figure 1C). Moreover, we also found that the higher the dose, the weaker the invasive and migrate ability (Figure 1D, E). All these indicated that demethylzeylasteral could inhibit the proliferation, invasion and migration of colorectal cells.

\section{Demethylzeylasteral inhibited the cell proliferation by causing cell cycle arrest and apoptosis}

In order to clarify the mechanism of its effect on the proliferation of colorectal cancer cells, we examined that whether demethylzeylasteral could affect the cell cycle arrest and apoptosis using the flow cytometry. What could be clearly seen was that the cell number in the G0/G1 phase increased, whereas decreased in S and G2/M phase in a dose-dependent manner (Figure 2A, B). Previously, it had been reported that cell cycle related proteins express in different periods. For example, $\mathrm{P}-\mathrm{Rb}$ and CCNB proteins tend to be highly expressed in $S$ or $G 2 / M$ phase $[18,19]$. As we supposed, compared with the control group, the protein levels of $\mathrm{P}-\mathrm{Rb}, \mathrm{CCNB}$ were lower in drug treatment group (Figure $3 \mathrm{C}$ ). As was showed in Figure 3A, with the increase of drug concentration, the apoptotic rate increased gradually. The apoptotic rate of control group was $6.4 \%$ in SW480; however, it was $23 \%$ when the drug concentration was $20 \mu \mathrm{M}$. Similar results were achieved in the RKO (Figure 3A, B). In addition, the apoptosis related protein levels of BCL-2, BAX and Cleaved-PARP were monitored by Western blot. After treatment with demethylzeylasteral, the protein expression of Cleaved-PARP, BAX were enhanced, and the level of BCL-2 was inhibited (Figure 3C). These results showed that the cell cycle arrest and apoptosis were involved in the cell proliferation inhibited by demethylzeylasteral.

\section{Demethylzeylasteral enhanced the chemo- sensitivity of human CRC cells to 5-FU}

As explained in the introduction, it was clear that 5-FU was the first choice for treatment of colorectal cancer. Therefore, we supposed that it may be more effective than single drug, when combined 5-FU with Demethylzeylasteral. Interesting, the combination 5-FU with Demethylzeylasteral demonstrated a higher inhibition rate compared with 5-FU monotherapy (Figure 4A, B). Furthermore, the inhibition rates of combination were significantly higher when demethylzeylasteral concentration increased. Notably, the most CDI values were below 1 in SW 480 when combined 5-FU with demethylzeylasteral, showing they function synergistically. And the most CDI values were close to 1 in RKO, suggesting they function additionally (Figure 4C). From Figure 4D, we could draw a conclusion that Demethylzeylasteral brought down the IC50 value of 5-FU. So we could get that Demethylzeylasteral enhance the chemosensitivity to 5-FU.

\section{The effect of 5-FU combined with demethylzeylasteral was more dramatic}

In order to verify whether the combination of the two drugs could really work better, we selected the appropriate concentration for the experiment. Figure $5 \mathrm{~A}$ revealed that there was a gradual decrease in the cell number that was treated with 5-FU and demethylzeylasteral. The evidences confirmed that demethylzeylasteral could enhance the anti-tumor effect of 5-FU in Colorectal cancer cells. The same result was verified by the clony experiment (Figure $5 B)$. It can be seen that there was a better effect when the two drugs were combined.

\section{The network pharmacological relationship between demethylzeylasteral and colorectal cancer}

We obtained the 3D structure of demethylzeylasteral from PubChem database (Figure 
6A). A total of 283 possible binding sites of demethylzeylasteral were predicted by PharmMapper database (Table S2). At the same time, we searched for a total of 10224 genes related to Colorectal cancer through TCGA database, which were differentially expressed genes between normal and neoplastic tissues (fold change $>1.4$ or $<0.7, P<0.05$ ) (Table S3). Compared with normal tissues, 6227 genes were up-regulated and 3997 genes were down-regulated in cancer tissues (Figure 6B). As shown in Figure 6C, we had a total of 150 genes which were associated with demethylzeylasteral and Colorectal cancer by constructing a Venn diagram (Table S4). We used cytoscape software to visualize the "Colorectal - targets - Demethylzeylasteral" network (Figure 6D). A PPI was analyzed by using String database. (Figure 7A, Table S5). In the PPI network, we found 15 hub genes (Figure 7B, Table S6). These genes may play an important role in the regulation of the whole PPI network. For example, EGFR, CCNA2 and CDK2 played important roles in cell cycle. Or MDM2 and BCL2L1 could affect the cell apoptosis. Morever, we also carried out the analysis of the survival rate of these hub genes in CRC through Oncolnc database. The cox regression results were shown in Figure S1. The genes, including AR, BCL2L1, EGFR, ESR1, were correlated negatively with overall survival. However, CCNA2, CHEK1, MDM2 played the opposite role.
A

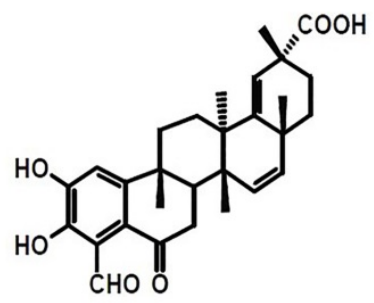

B

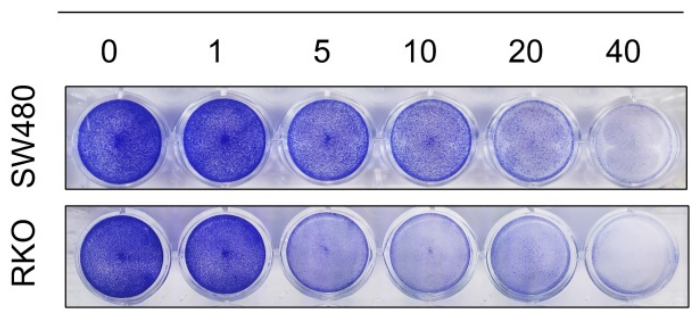

C

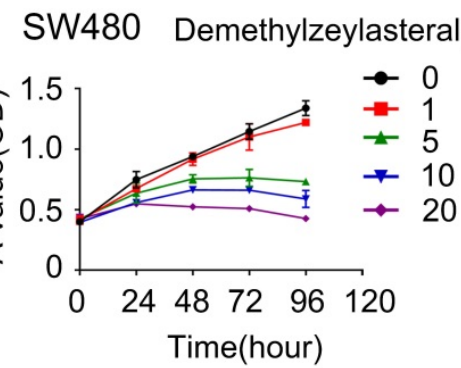

RKO Demethylzeylasteral $(\mu \mathrm{M})$

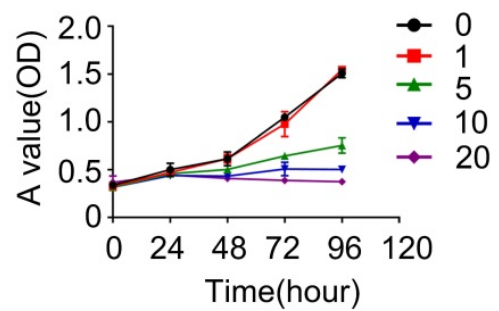

D
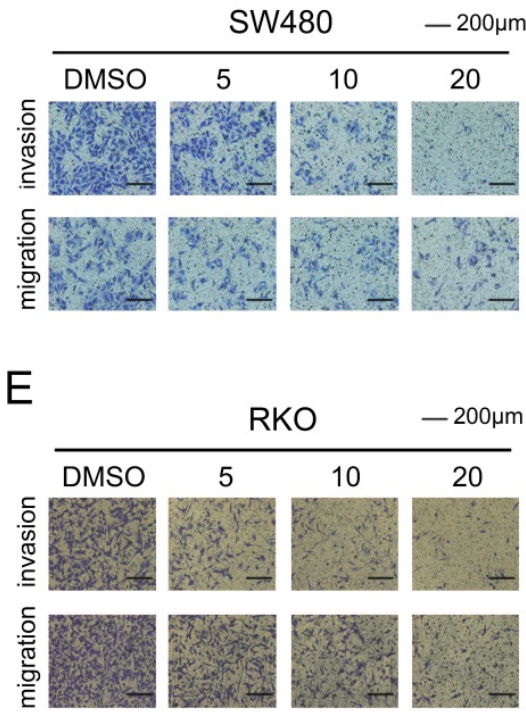
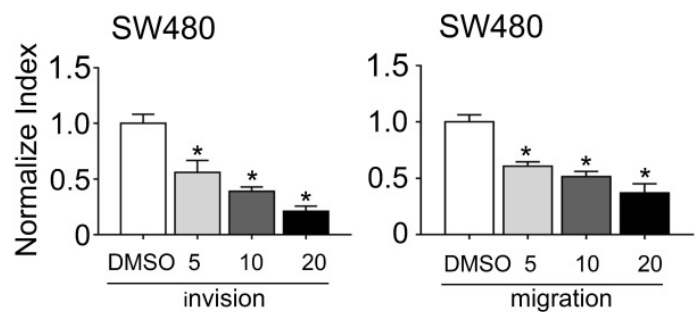

RKO

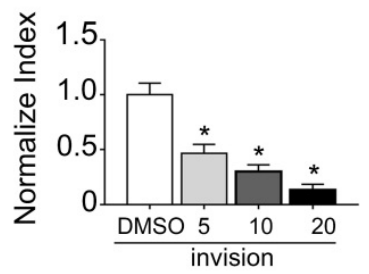

RKO

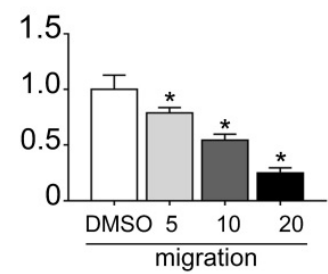

Figure 1. The effect of Demethylzeylasteral on the growth, invasion and migration of colorectal cancer cells. A. The chemical structural formula of demethylzeylasteral. B. SW480 and RKO were treated for 48 hours by Demethylzeylasteral of gradient concentrations. Colony formation experiments had been performed. The darker the colour, the more the cells. C. SW480 and RKO were treated by the concentration gradient of demethylzeylasteral. The cell viability was measured by MTS assay. Statistics of MTS experiments of SW480 and RKO were shown in Table S1. D and E. SW480 and RKO were treated by demethylzeylasteral of gradient concentrations. The invasion and migration activity were detected by transwell experiments. The right is the statistical chart. $* P<0.001$. 
A
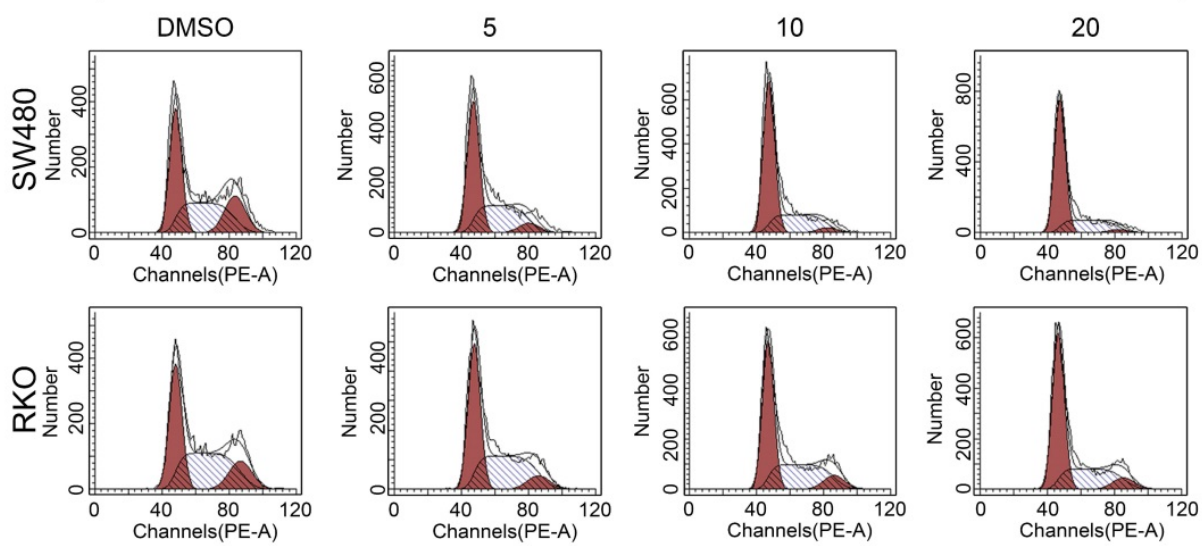

$\mathrm{B}$
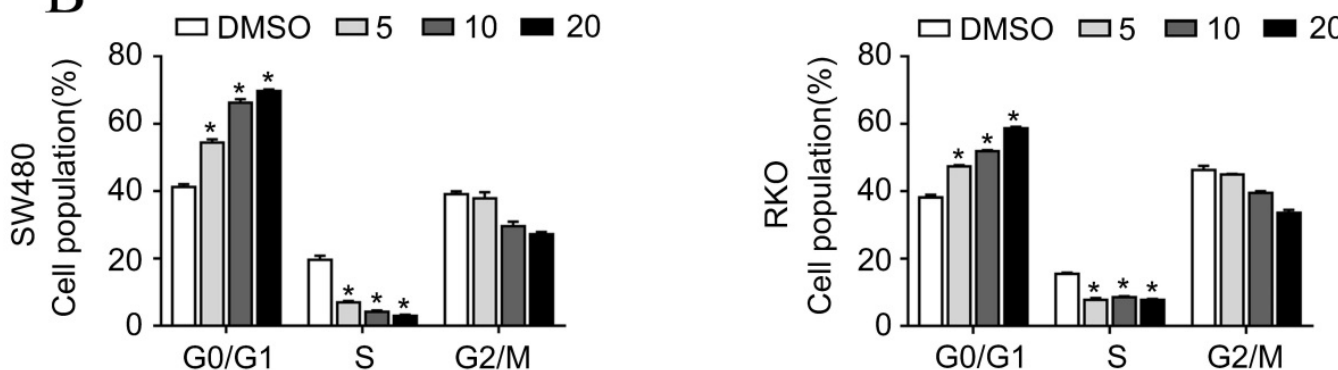

Figure 2. The effect of demethylzeylasteral on the cell cycle of colorectal cancer cells. A. SW480 and RKO were treated for 24 hours by demethylzeylasteral of gradient concentrations. Cell cycle was detected by flow cytometry. B. Statistical analysis on chart A.

A

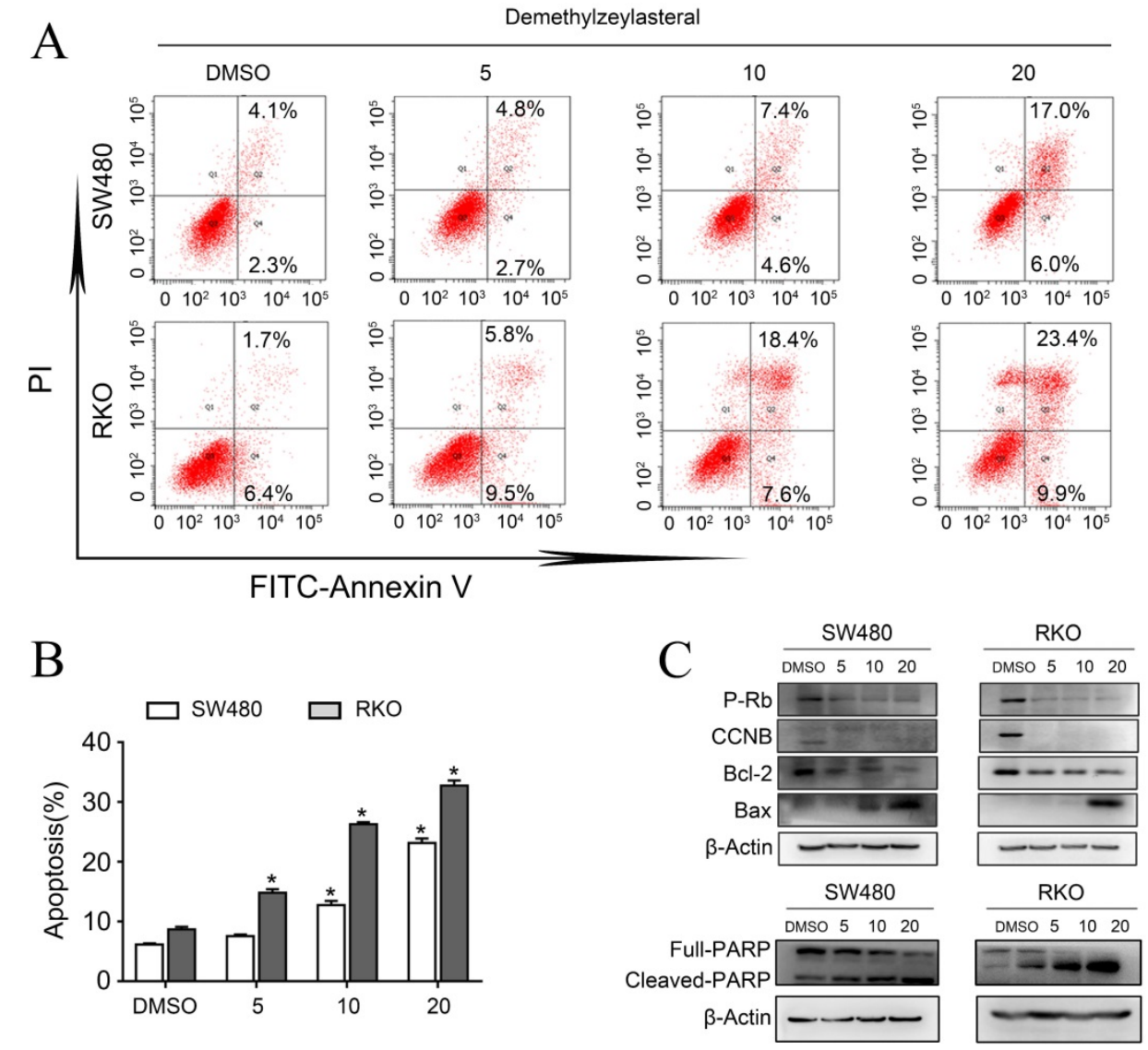

emethylzeylasteral

Figure 3. The effect of demethylzeylasteral on the apoptosis of colorectal cancer cells. A. SW480 and RKO were treated for $24 \mathrm{~h}$ by demethylzeylasteral of gradient concentrations. Apoptosis was detected by flow cytometry. B. Statistical chart of A chart. C. SW 480 and RKO were treated for $24 \mathrm{~h}$ by demethylzeylasteral of gradient concentrations. The protein expression of BAX, BCL-2, CCNB, P-Rb and Cleaved-PARP were detected by Western blot. $* P<0.001$. 
A

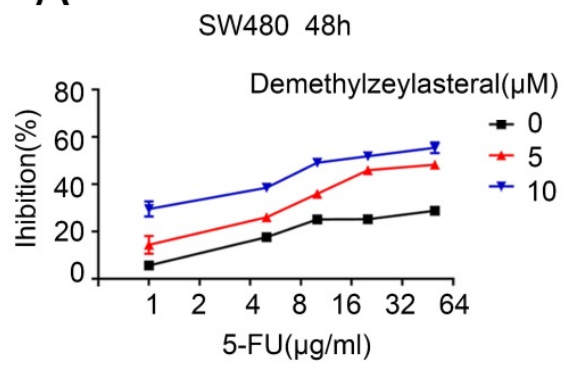

C

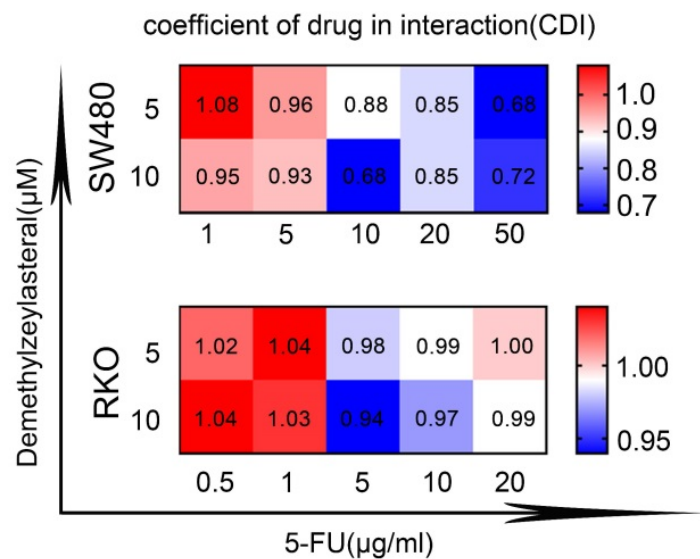

B

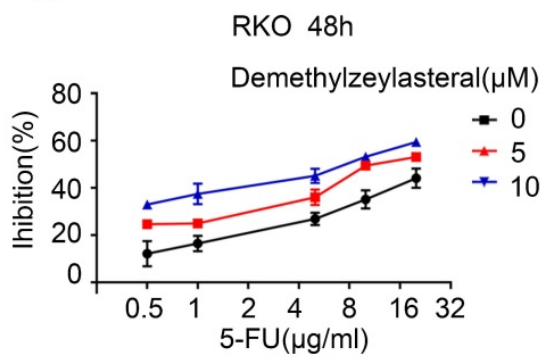

D

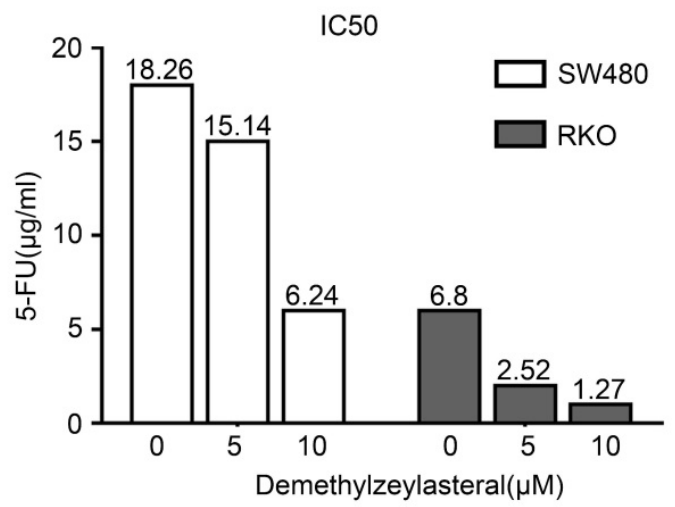

Figure 4. Demethylzeylasteral enhanced sensitivity of Colorectal cancer cells to 5-FU. A and B. SW480 and RKO were treated by demethylzeylasteral and 5-FU of gradient concentrations. The inhibition rates of colorectal cancer cells were tested by MTS assay. C. SW480 were treated for $60 \mathrm{~h}$ by demethylzeylasteral and 5 -FU of gradient concentrations. RKO were treated for $24 \mathrm{~h}$ by demethylzeylasteral and 5-FU of gradient concentrations. The interaction coefficient of the two drugs was calculated. CDI $>1$ antagonism, $\mathrm{CDI}=1$ addition, $\mathrm{CDI}<1$ synergism. D. SW480 were treated for $90 \mathrm{~h}$ by demethylzeylasteral and 5 -FU of gradient concentrations. RKO were treated for 60 hours by demethylzeylasteral and 5-FU of gradient concentrations. The IC50 values of 5-FU were calculated when combined with demethylzeylasteral.

A

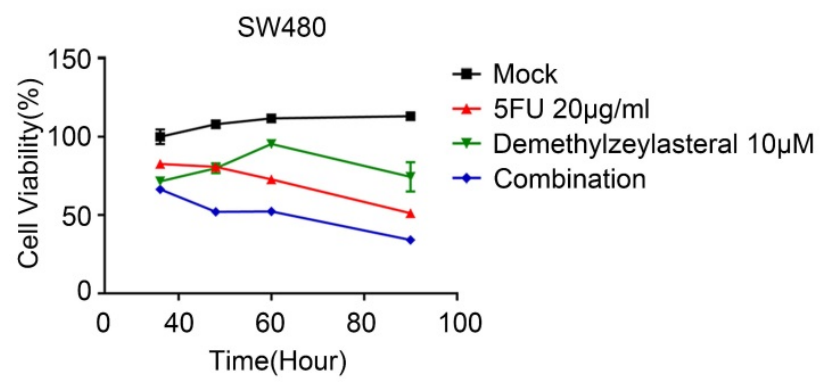

RKO

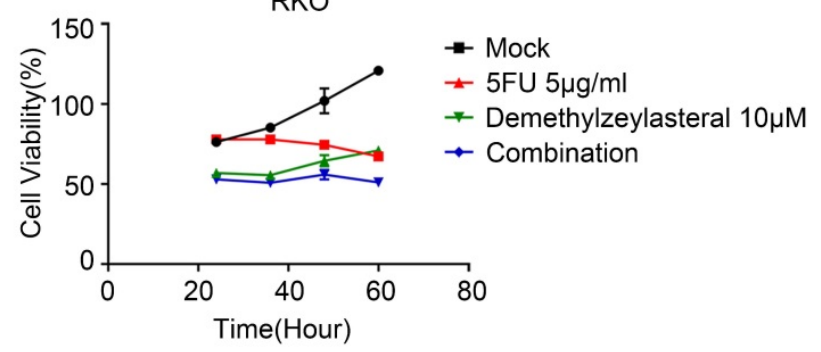

B

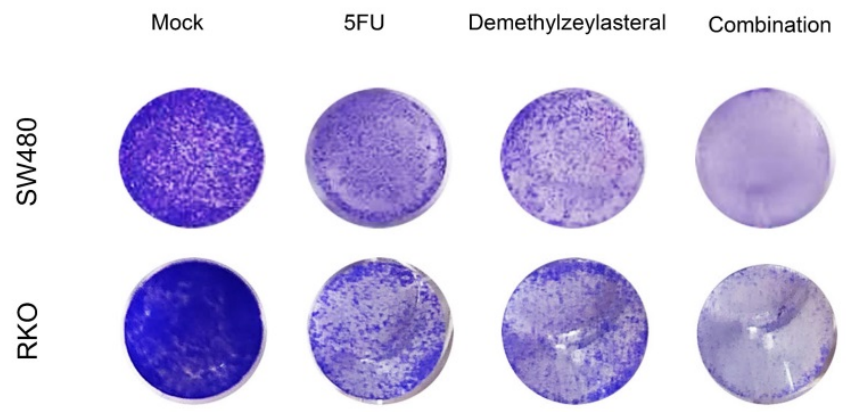

Figure 5. Combined therapeutic effect of demethylzeylasteral and 5-FU on colorectal cancer cells. SW480 and RKO were treated with demethylzeylasteral and 5 -FU. The concentration of 5-FU was $20 \mu \mathrm{g} / \mathrm{ml}$ in SW480, while it was $5 \mu \mathrm{g} / \mathrm{ml}$ in RKO. The cell toxicity was tested by MTS assay. B. SW480 and RKO were treated with demethylzeylasteral and 5-FU. Colony formation experiments had been performed. 
A
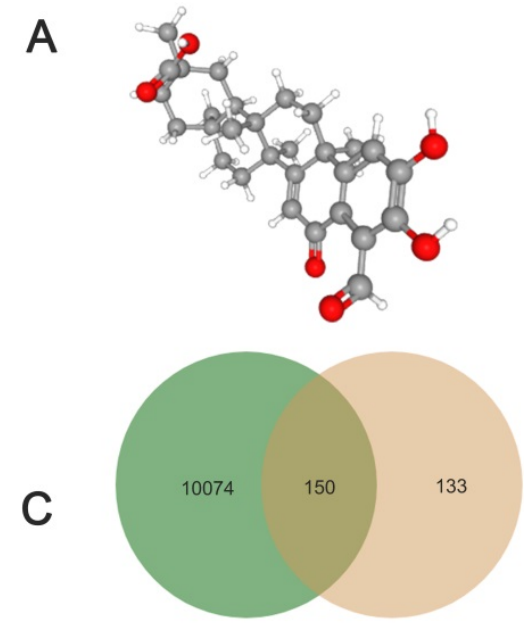

B

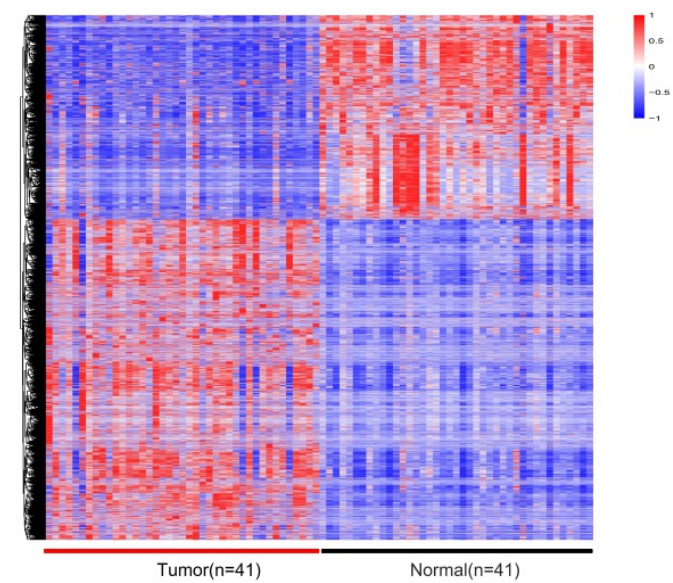

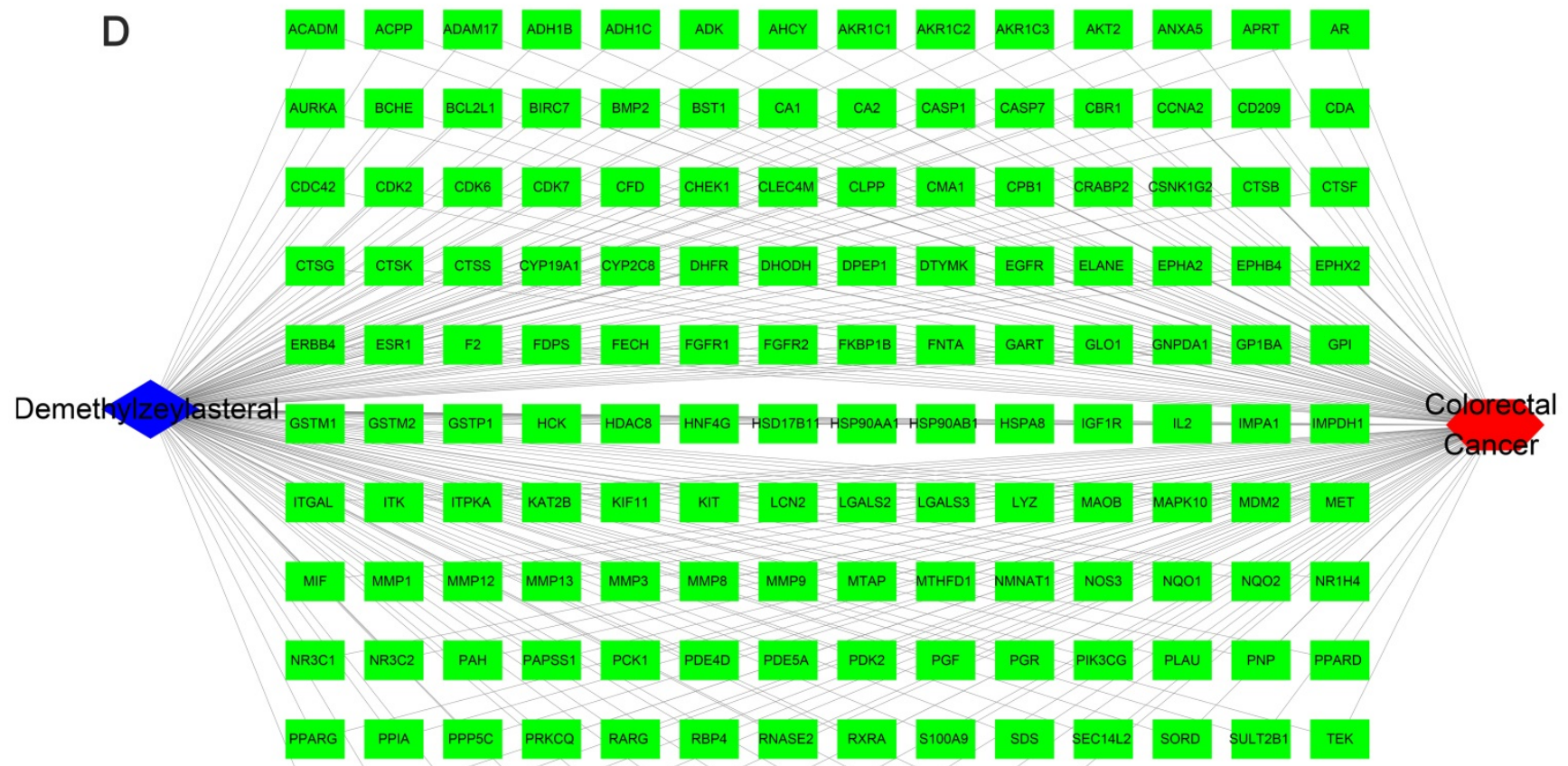

\begin{tabular}{llllllllll}
\hline THRB & TK1 & TNK2 & TPH1 & TPSB2 & TREM1 TYMS & VDR & WAS & YARS \\
\hline
\end{tabular}

Figure 6. The network pharmacological relationship between demethylzeylasteral and colorectal cancer. A. The 3D molecular structure of Demethylzeylasteral was derived from PubChem database. B. The heat map of differentially expressed genes in colorectal cancer tissue and normal tissue from TCGA database. C. A Venn diagram was constructed to show the relationship between colorectal cancer and demethylzeylasteral. Green colour represented colorectal cancer-related genes, and pink colour represented predicted binding genes. D. The "Colorectal - targets - Demethylzeylasteral" network.

\section{The GO and KEGG pathway enrichment analysis of 150 genes related to demethylzeylasteral and Colorectal cancer}

We performed GO and KEGG pathway enrichment analysis of these 150 genes by using DAVID database. The results showed that these 150 genes were mainly related to cytosol, extracellular region, cell surface, cell-cell junction, nuclear chromatin, mitochondrion, lysosome, or spindle microtubule (Figure 8B, Table S7). At the same time, they had the function of receptor, enzyme activity and so on (Figure 9A, Table S8). They were involved in the regulation of apoptotic process, regulation of cell proliferation, response to drug, glucose metabolic, immune response or regulation of inflammatory response (Figure 8A, Table S9). KEGG pathway analysis showed that these genes were enriched in many cancer-related pathways, such as PI3K-Akt signaling pathway, Ras signaling pathway, metabolic pathways, cell cycle, VEGF signaling pathway, apoptosis and so on (Figure 9B, Table S10). This suggested that demethylzeylasteral may affect colorectal cancer through these pathways. This provided a theoretical basis for our future research. 


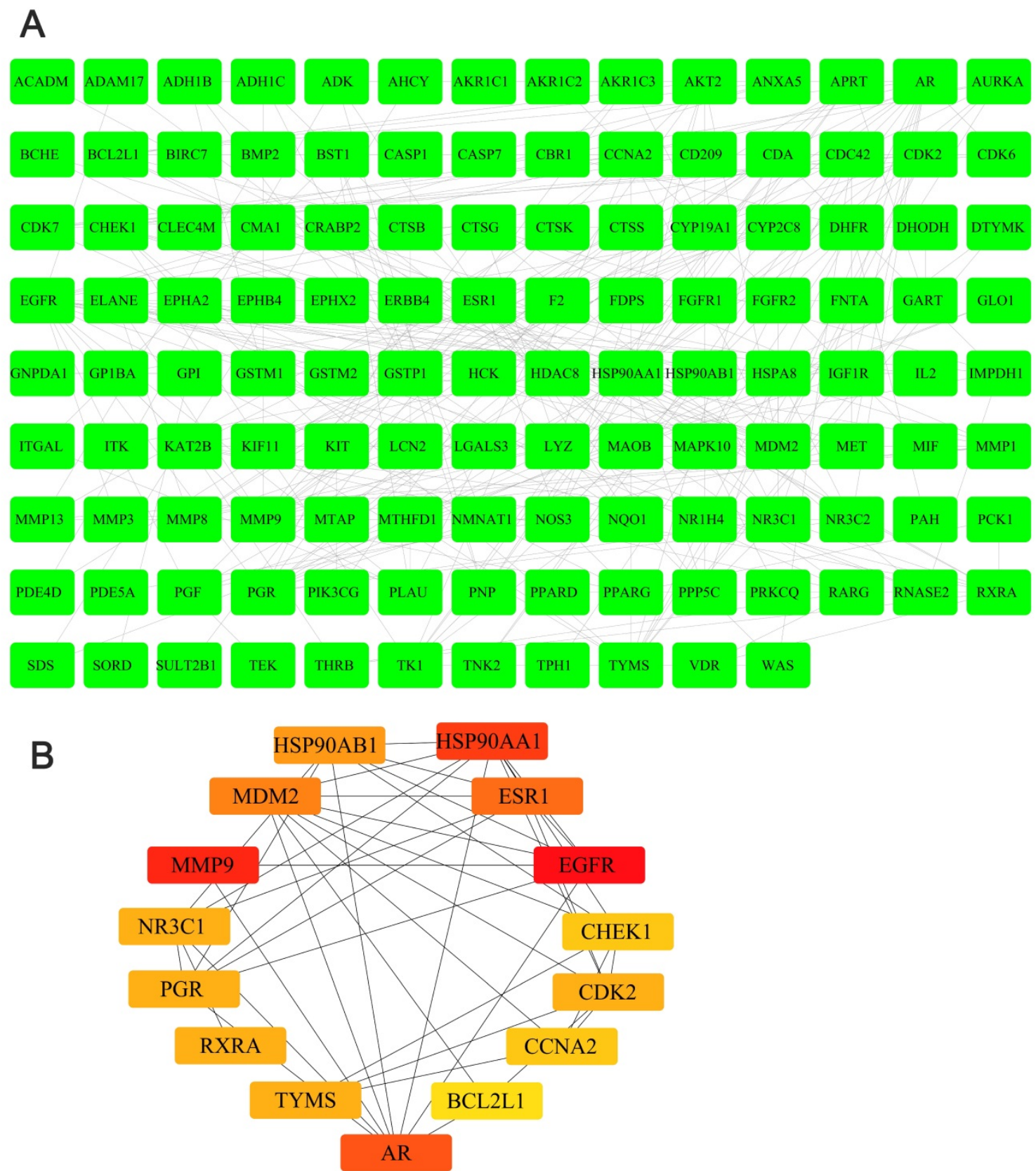

Figure 7. The PPI analysis and hub genes in PPI network. A. The PPI analysis of 150 intersection genes from figure $6 \mathrm{C}$ (interaction score $>0.7$ ). B. The hub genes in PPI network.

\section{Discussion}

Demethylzeylasteral was firstly found to have an effect on immunosuppressive and anti-inflammatory. Previous studies also had shown that demethylzeylasteral was involved in anti-cancer. Up to now, demethylzeylasteral had been reported to have an anti-cancer effect in breast cancer, glioma and so on. However, there was no study that demethylzeylasteral had an anti-cancer effect in colorectal. In our study, we indicated that demethylzeylasteral could inhibit cell viability in dosedependent manner. Forever, demethylzeylasteral was found to cause cell cycle arrest and apoptosis in colorectal. Our research showed that demethylzeylasteral may have the potential to be applied to clinical trials in the future.

Colorectal was one of the most common cancers in China. In recent decades, 5-FU had been used as a first-line treatment for colorectal cancer [20]. Nevertheless, when used as a single drug, the nonresponse rate for 5 -FU was about $50 \%$ for metastatic Colorectal cancer patients [21, 22]. Hence, it was crucial to enhance chemosensitivity of 5-FU.

5-FU and gemcitabine belong to Pyrimidine analogues and could cause the cell apoptosis. Also, a 
present study indicated that Demethylzeylasteral could enhance PC cells' chemosensitivity to gemcitabine. But it was unknown that whether demethylzeylasteral could enhance the coloerectal cells' chemosensitivity to 5-FU. Based on these, we supposed that demethylzeylasteral may potentiate the activity of 5-FU. Followed, we verified that demethylzeylasteral can decrease the IC50 value of 5 -FU and showed the combination demethylzeylasteral and 5-FU could achieve a better therapeutic effect, compared with single drug. Here, we carried out for the first time that demethylzeylasteral could enhance the coloerectal cells' chemosensitivity to $5-\mathrm{FU}$.
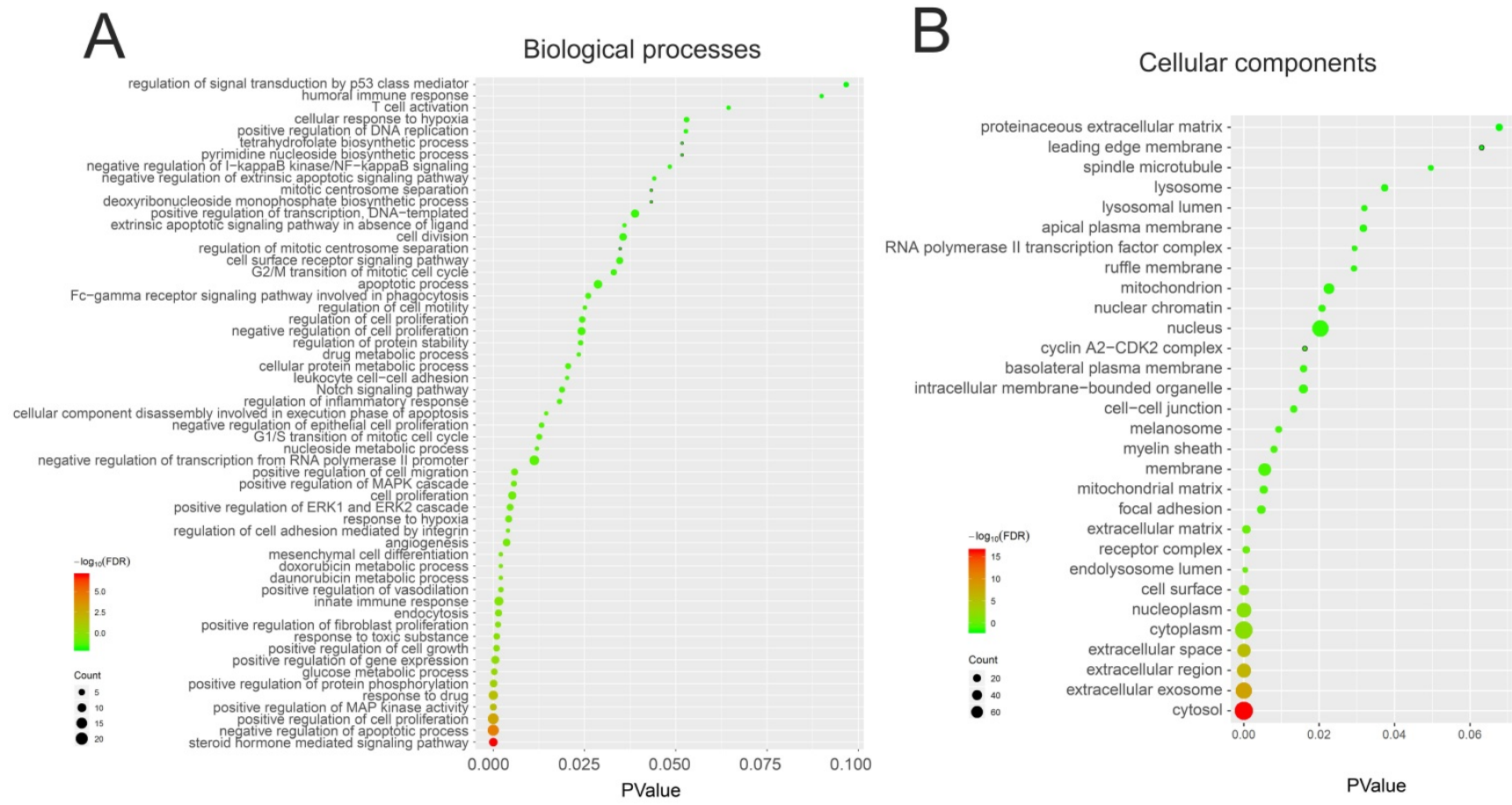

Figure 8. The GO analysis of 150 genes related to Demethylzeylasteral and Colorectal cancer. A. Biological processes. B. Cellular components.
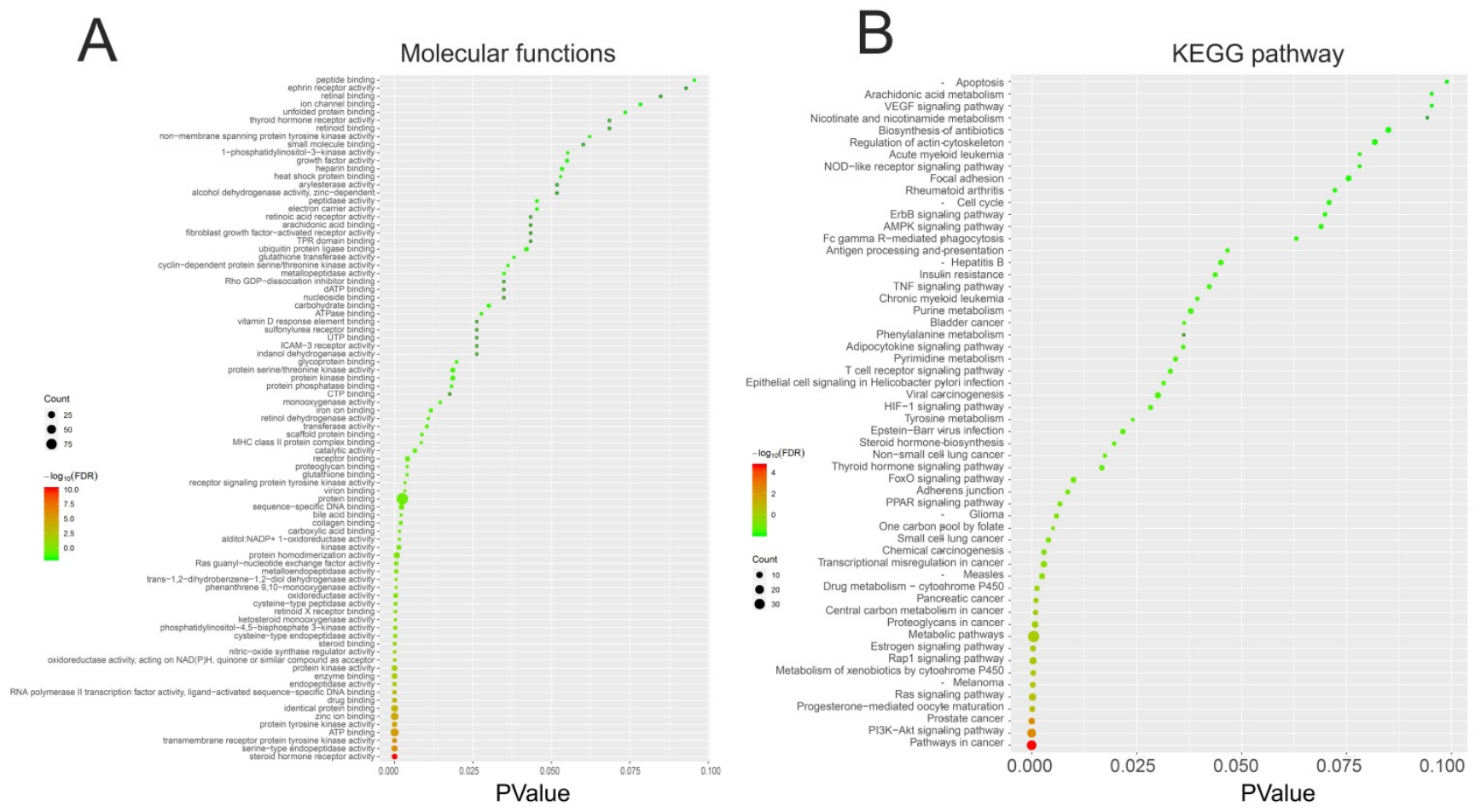

Figure 9. The GO and KEGG pathway enrichment analysis of 150 genes related to Demethylzeylasteral and Colorectal cancer. A. Molecular functions. B. KEGG pathway analysis. 
In order to explore the potential mechanism of the effect of demethylzeylasteral on colorectal cancer cells, we predicted the possible target genes of demethylzeylasteral and got the genes related to colorectal cancer. Followed, we took the intersection of the above two types of genes. We thought that these intersection genes may play important roles in the mechanism of demethylzeylasteral on colorectal cancer cells. To more intuitively show the relationship among these intersection genes, demethylzeylasteral and colorectal cancer, we carried out a "Colorectal targets - Demethylzeylasteral" network. After that, the PPI network of these intersection genes was constructed through String database and 15 hub genes were found (EGFR, MMP9, HSP90AA, AR, ESR1, MDM2, HSP90AB1, TYMS, CDK2, RXRA, PGR, NR3C1, CCNA2, CHEK1, BCL2L1). Many genes were related to overall survival. We believed that these 15 genes were key points in the regulation of the whole PPI network. We found that some genes were related to the proliferation and apoptosis of Colorectal cancer, such as EGFR, CDK2, CCN2 and BCL2L1 [23-26], and some were related to the prediction of chemotherapy for colorectal cancer, such as EGFR, TYMS [27, 28]. Others, such as MMP9 [29], were associated with the metastasis of colorectal cancer. These phenomena were consistent with our experimental results and our pathway analysis. As our experimental results shown, demethylzeylasteral could promote apoptosis, inhibit proliferation and inhibit metastasis. GO enrichment analysis showed that these genes may affect cell cycle, apoptosis and invasion. KEGG pathway analysis showed that many cancer-related pathways were enriched. Among them, the PI3k-AKT pathway (ranked first in KEGG pathways) was officially the downstream pathway of EGFR [30] (ranked first in hub genes). In addition, many previous studies had reported that demethylzeylasteral were associated with proliferation, apoptosis and invasion in other cancers, which were consistent with the function of demethylzeylasteral verified by us in Colorectal cancer.

Finally, we carried out GO and KEGG pathway analysis. GO enrichment analysis showed that these genes may affect cell cycle, apoptosis and invasion, in turn verifying our experimental results. KEGG pathway analysis showed that many cancer-related pathways were enriched. This provided a theoretical basis for our future research.

Now, we had noted the key role that demethylzeylasteral played in colorectal cells. Further research would be conducted to verify that whether a consistent result could be observed in vivo. In addition, it was essential to explore the toxicity of demethylzeylasteral in vivo. Our study may represent a new therapeutic strategy to overcome the chemotherapeutic resistance in CRC.

\section{Abbreviations}

CRC: Colorectal cancer; 5-FU: 5-fluorouracil; GO: Gene Ontology; KEGG: Kyoto Encyclopedia of Genes and Genomes; TCGA: The Cancer Genome Atlas database.

\section{Supplementary Material}

Supplementary figure and tables.

http://www.jcancer.org/v11p6059s1.pdf

\section{Acknowledgments}

This work is supported by the Special Fund for Marathon (NCC2017A23).

\section{Competing Interests}

The authors have declared that no competing interest exists.

\section{References}

1. Inadomi J, Jung B. Colorectal Cancer-Recent Advances and Future Challenges. Gastroenterology. 2020; 158: 289-290.

2. Bray F, Ferlay J, Soerjomataram I, et al. Global cancer statistics 2018: GLOBOCAN estimates of incidence and mortality worldwide for 36 cancers in 185 countries. CA Cancer J Clin. 2018; 68: 394-424.

3. Wang X, Mo F-M, Bo H, et al. Upregulated Expression of Long Non-Coding RNA, LINC00460, Suppresses Proliferation of Colorectal Cancer. J Cancer. 2018; 9: 2834-2843.

4. Dekker E, Tanis PJ, Vleugels JLA, et al. Colorectal cancer. Lancet . 2019; 394: $1467-1480$

5. Zhu J, Tan Z, Hollis-Hansen K, et al. Epidemiological Trends in Colorectal Cancer in China: An Ecological Study. Dig Dis Sci. 2017; 62: 235-243.

6. Wang YN, Zeng ZL. CPT1A-mediated fatty acid oxidation promotes Colorectal cancer cell metastasis by inhibiting anoikis. Oncogene. 2018; 37: 6025-6040.

7. Miyamoto $\mathrm{Y}$, Oki E, Saeki $\mathrm{H}$, et al. Recent Advances in Systemic Chemotherapy for Metastatic Colorectal Cancer. Gan To Kagaku Ryoho. 2016; 43: 15-23.

8. Marin-Vicente C, Lyutvinskiy $Y$, Romans Fuertes $P$, et al. The effects of 5-fluorouracil on the proteome of colon cancer cells. J Proteome Res. 2013; 12: 1969-1979.

9. Vodenkova $\mathrm{S}$, Buchler $\mathrm{T}$, Cervena $\mathrm{K}$, et al. 5-fluorouracil and other fluoropyrimidines in Colorectal cancer: Past, present and future. Pharmacol Ther. 2020; 206: 107447-107447.

10. van der Stok EP, Spaander MCW, Grünhagen DJ, et al. Surveillance after curative treatment for Colorectal cancer. Nat Rev Clin Oncol. 2017; 14: 297-315.

11. $\mathrm{Hu} \mathrm{Q}$, Yang C, Wang $\mathrm{Q}$, et al. Demethylzeylasteral (T-96) Treatment Ameliorates Mice Lupus Nephritis Accompanied by Inhibiting Activation of NF-kB Pathway. PLoS One. 2015; 10: e0133724-e0133724.

12. Li L, Ji Y, Fan J, et al. Demethylzeylasteral (T-96) inhibits triple-negative breast cancer invasion by blocking the canonical and non-canonical TGF- $\beta$ signaling pathways. Naunyn Schmiedebergs Arch Pharmacol. 2019; 392: 593-603.

13. Zhang K, Fu G, Pan G, et al. Demethylzeylasteral inhibits glioma growth by regulating the miR-30e-5p/MYBL2 axis. Cell Death Dis. 2018; 9: 1035-1035.

14. Zhao Y, He J, Li J, et al. Demethylzeylasteral inhibits cell proliferation and induces apoptosis through suppressing MCL1 in melanoma cells. Cell Death Dis. 2017; 8: e3133-e3133

15. Wang F, Tian X, Zhang Z, et al. Demethylzeylasteral (ZST93) inhibits cell growth and enhances cell chemosensitivity to gemcitabine in human pancreatic cancer cells via apoptotic and autophagic pathways. Int J Cancer. 2018; 142: 1938-1951.

16. Zhou W, Sun G, Zhang Z, et al. Proteasome-Independent Protein Knockdown by Small-Molecule Inhibitor for the Undruggable Lung Adenocarcinoma. J Am Chem Soc. 2019; 141: 18492-18499.

17. Liu YG, Zheng XL, Liu FM. The mechanism and inhibitory effect of recombinant human P53 adenovirus injection combined with paclitaxel on human cervical cancer cell HeLa. Eur Rev Med Pharmacol Sci. 2015; 19: 1037-1042. 
18. Zhang $\mathrm{T}$, Zhou $\mathrm{Y}, \mathrm{Li} \mathrm{L}$, et al. CenpH regulates meiotic $\mathrm{G} 2 / \mathrm{M}$ transition by modulating the APC/CCdh1-cyclin B1 pathway in oocytes. Development. 2017; 144: 305-312.

19. Chung K-S, Hong JY, Lee J-H, et al. $\beta$-Caryophyllene in the Essential Oil from Chrysanthemum Boreale Induces G(1) Phase Cell Cycle Arrest in Human Lung Cancer Cells. Molecules. 2019; 24: 3754-3754.

20. He J, Pei $\mathrm{L}$, Jiang $\mathrm{H}$, et al. Chemoresistance of Colorectal cancer to 5-fluorouracil is associated with silencing of the BNIP3 gene through aberrant methylation. J Cancer. 2017; 8: 1187-1196.

21. Douillard JY, Cunningham D, Roth AD, et al. Irinotecan combined with fluorouracil compared with fluorouracil alone as first-line treatment for metastatic Colorectal cancer: a multicentre randomised trial. Lancet. 2000; 355: 1041-1047.

22. Giacchetti S, Perpoint B, Zidani R, et al. Phase III multicenter randomized trial of oxaliplatin added to chronomodulated fluorouracil-leucovorin as first-line treatment of metastatic Colorectal cancer. J Clin Oncol. 2000; 18: 136-147.

23. Ge H, Yan Y, Yue C, et al. Long Noncoding RNA LINC00265 Targets EGFR and Promotes Deterioration of Colorectal Cancer: A Comprehensive Study Based on Data Mining and in vitro Validation. Onco Targets Ther. 2019; 12: 10681-10692.

24. Chen MJ, Cheng AC, Lee MF, et al. Simvastatin induces G1 arrest by up-regulating GSK3 $\beta$ and down-regulating CDK4/cyclin D1 and CDK2/cyclin E1 in human primary colorectal cancer cells. J Cell Physiol. 2018; 233: 4618-4625.

25. Wang T, Zhang WS, Wang ZX, et al. RAPTOR promotes colorectal cancer proliferation by inducing mTORC1 and upregulating ribosome assembly factor URB1. Cancer Med. 2020; 9: 1529-1543.

26. Healy ME, Boege Y, Hodder MC, et al. MCL1 is Required for Maintenance of Intestinal Homeostasis and Prevention of Carcinogenesis in Mice. Gastroenterology. 2020; S0016-5085.

27. Srivatsa S, Paul MC, Cardone $C$, et al. EGFR in Tumor-Associated Myeloid Cells Promotes Development of Colorectal Cancer in Mice and Associates With Outcomes of Patients. Gastroenterology. 2017; 153: 178-190.

28. Xu F, Ye ML, Zhang YP, et al. MicroRNA-375-3p enhances chemosensitivity to 5 -fluorouracil by targeting thymidylate synthase in Colorectal cancer. Cancer Sci. 2020; 111: 1528-41.

29. Peng K, Su G, Ji J, et al. Histone demethylase JMJD1A promotes colorectal cancer growth and metastasis by enhancing $W n t / \beta$-catenin signaling. J Biol Chem. 2018; 293: 10606-10619.

30. Wang Y, Wang W, Wu X, et al. Resveratrol Sensitizes Colorectal Cancer Cells to Cetuximab by Connexin 43 Upregulation-Induced Akt Inhibition. Front Oncol. 2020; 10: 383-383. 